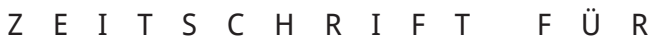

$\begin{array}{lllllllllllllllllllll}V & E & R & W & A & L & T & U & N & G & S & G & E & S & C & H & I & C & H & T & E\end{array}$

$\begin{array}{lllllllll}B & A & N & D & 4 & 2 & 0 & 1 & 9\end{array}$

S E I I

D O I : $10.2478 / \mathrm{ADH}$ I- $2019-0012$

\title{
Wieder gelesen: Cornelia Vismann, "Akten « (2000)
}

\section{FELIX LÜTTGE}

"Sind die Akten des Staates vollständig, dann sind sie langweilig. Fehlt auch nur eine Seite, dann ist ein Verbrechen geschehen. ${ }^{1}$

\section{Y2K}

Die beiden nächstliegenden historischen Einordnungen, um die man fast 20 Jahre nach Erscheinen der "Akten" nicht herumkommt, nimmt Cornelia Vismann selbst vor. Das Zeitalter papierner Akten schien im Jahr 2000 an sein Ende gekommen, der Millennium-Bug hatte keinen nennenswerten Schaden angerichtet. Die Geschichte, die Vismanns "Akten. Medientheorie und Recht « erzählt, ist die eines "im Aussterben begriffene[n] Medium[s] «2: Aus Canzleyen sind Computer geworden, deren CPU die Registerpolitik des Stauferkaisers Friedrich II. fortsetzen und auf deren Bildschirmen Akten nur noch als icons auftauchen und files heißen. Nicht umsonst hat Vismann das Buch »auch für diejenigen geschrieben, die schon nicht mehr mit physisch-realen Akten zu tun haben «. ${ }^{3}$ Cornelia Vismanns Akten ist ein Text, der weiß, dass er ein Datum hat, und das gilt medientechnisch ebenso wie -theoretisch.
"Eine in Berlin erprobte Medientheorie im Umkreis von Friedrich Kittler « ${ }^{4}$ nennt Vismann das Projekt jenes Denkkollektivs, als dessen Teil sie ihre Dissertation in den Neunzigerjahren schrieb und das heute pejorativ 'Kittlerjugend`, in der Selbstbeschreibung eines Lehrstuhls für Medientheorien >Berliner Schule und im anglophonen Wissenschaftsraum `German Media Theory heißt. Diese intellektuelle Herkunft liest man unschwer aus dem Text heraus, was Valentin Groebner Anlass gab, in seiner Rezension für die "Neue Zürcher Zeitung « einen >Generationsjargon`zu beklagen. ${ }^{5}$ Als zehn Jahre später die "Akten« einer ersten Relektüre unterzogen wurden, sah Mario Wimmer im fröhlichen Gebrauch französischen Theorievokabulars nicht bloß die Manierismen einer in der Berliner Sophienstraße betriebenen Kulturwissenschaft, sondern einen "Denkstil, der Gebrauch vom Computer- und Maschinenvokabular der Kybernetik macht, um damit historische Phänomene $\mathrm{zu}$ analysieren. $\aleph^{6}$ Vismanns Lacanismen mögen einem heute vor allem wie ein zeitlicher Index vorkommen, aber einen solchen $\mathrm{zu}$ besitzen, gehört zu den Eigenschaften von Denkstilen. Wer froh ist, dass so heute keiner mehr schreibt, müsste ebenso froh sein, dass auch solche Bücher nicht mehr geschrieben werden. 
Der medientheoretische Kontext führt, wie könnte es anders sein, auf den medientechnischen zurück. Friedrich Kittler nannte schon im Sommersemester 1988 an der Ruhr-Universität Bochum in einem Seminar über "Lacan, Foucault, Derrida" - also über die für Vismanns Studie maßgeblichen Autoren - die "allgemeine, d. h. computerisierte Datenverarbeitung " das historische Apriori des Poststrukturalismus. ${ }^{7}$ Später wird er sich dafür auf einen Besuch Derridas in Siegen berufen, der den Computer zur Voraussetzung der Dekonstruktion erklärte. ${ }^{8}$ Es lohnt sich, daran zu denken, wenn man Vismanns "grammatologische Betrachtung der Akten" wiederliest. ${ }^{9}$ "Die poststrukturalistischen Programme sind nicht geschrieben, um referierbar zu werden. Wirksamer ist es, sie ins Spiel zu bringen «, ist noch so ein Satz Kittlers, der eben nicht nur seine, sondern auch Vismanns theoretische Vorgehensweise auf den Computer zurückführt. ${ }^{10}$ Dass aber der Computer ein System ist, "das Zeichen manipulieren kann, ohne Rücksicht auf einen hermeneutischen Sinn zu nehmen «, macht ihn zur "methodische[n] Vergleichsgrundlage sowohl für die Lektürepraxis der Poststrukturalisten als auch für die Arbeitsweise einer Medienwissenschaft in der Tradition Kittlers« - und verbindet ihn ebenso mit den autorlosen, von nicht-syntaktischen Zeichen wie $W V, z K$ oder $z U m l$ gesteuerten Akten, die Cornelia Vismann gerade nicht daraufhin befragt, was sie sind, sondern wie sie funktionieren. ${ }^{11}$ Während Kittlers eigene Erzählung der Mediengeschichte, die den Computer so deutlich als eigene Möglichkeitsbedingung ausweist, im Computer an ihr Ende kommt, nehmen Vismanns "Akten" davon ihren Ausgang, auch ohne von ihm zu handeln: als Geschichte sichtbarer Zeichen und beschreibbarer Praktiken, die als vermeintlich verborgene Codes oder Gesetze die Black Boxes digitaler Verwaltung organisieren. "Die Geschichte der Akten«, schließt Vismanns Studie, »enthält [...] auch eine Vorgeschichte des Computermediums. Nicht, weil bewusst alte Aktenpraktiken auf das neue Medium übertragen werden würden, sondern weil in die sich ihrer historischen Dimension unbewusste Organisation digitaler Hardware administrative Techniken vergangener Jahrhunderte eingeschrieben sind in Form von stacks, files, compiler oder registers. Mit diesem medienarchäologischen Rückbezug auf >Akten` würde hat. «12 So ist Vismanns Buch auch ein Beispiel für Kulturtechnikforschung. ${ }^{13}$

\section{Dr. iur. Cornelia Vismann}

Das ist ungewöhnlich für ein juristisches Buch. Cornelia Vismann war Juristin. "Akten. Medientechnik und Recht" ist die Dissertation, die sie nach einem Studium des Rechts und der Philosophie zwar in Berlin geschrieben, aber in Frankfurt am Main eingereicht und verteidigt hat. Und so verdankt dieses Buch die andere Hälfte der Leichtigkeit, mit der es Disziplingrenzen überschreitet, den Rechtswissenschaftlern Michael Stolleis und Günter Frankenberg wie wohl vor allem der 2008 verstorbenen Rechtshistorikerin Marie Theres Fögen, die Vismann 2002 ans Max-Planck-Institut für europäische Rechtsgeschichte geholt hatte. Wie ihre Dissertation wechselte auch Cornelia Vismann während und nach der Promotion zwischen den Fakultäten hin und her und saßs später zwischen allen Lehrstühlen, bis sie schließlich nach einer juristischen Habilitation über die Verfassung nach dem Computer und mit einer venia legendi für öffentliches Recht auf eine Professur für Geschichte und Theorie der Kulturtechniken an der Bauhaus-Universität Weimar berufen wurde. Kurz danach wurde sie krank. Cornelia Vismann starb 2010. Die Akten blieben die einzige zu ihren Lebzeiten publizierte Monografie.

\section{Grammatologie der Akten}

Die Akten in "Akten« entziehen sich einer allgemeinen Definition. Sie sind keine abzählbar-diskreten Einheiten, sind nicht nur der Inhalt von LeitzOrdnern oder Hängeregistraturen. Die Akten, von denen Vismann schreibt, "können in verschiedenen Formationen vorkommen: als lose Blätter, in Kästchen liegend, mit Packpapier umwickelt oder in Kapseln verschlossen, als ein mit Faden verschnürtes Bündel oder als aufrecht stehender Ordner, der alles aufnimmt, was zwischen zwei Pappdeckeln Platz hat «. ${ }^{14}$ Erhard Schüttpelz charakterisiert sie ausgehend von Vismann als Verkettung von Verwaltungsoperationen: "Eine Akte ist ein Akt ist die Akte des Akts und der Akt der 
Akte «. ${ }^{15}$ Oder, Cornelia Vismann: Akten »enthalten sich selbst als Ablauf «. ${ }^{16}$ Sie sind "Variablen im Universum der Schrift und des Rechts" und deshalb nur formal zu definieren als "das, was einen bestimmten Typ von Recht generiert «. ${ }^{17}$ So gilt Vismanns Aufmerksamkeit den medientechnischen Bedingungen, unter denen Aufzeichnungen $\mathrm{zu}$ Akten werden, und den von diesen Akten gesteuerten Formalisierungen und Ausdifferenzierungsprozessen des Rechts.

Auch das Recht definiert Vismann in Abhängigkeit von Akten. Es ist ihr nicht Instrument oder Medium der Konfliktentscheidung, sondern "Formenreservoir autoritativer und administrativer Handlungen, das sich wiederum in Akten konkretisiert ${ }^{18}{ }^{18}$ Was Recht ist, steht in Akten, und was in Akten steht, wird Recht. Das ist nicht tautologisch, sondern historisch-medienmaterialistisch: Recht ist bei Vismann nicht einfach System, Konstante oder Tradition, sondern eine historisch spezifische Konstellation, abhängig von medientechnologischen Bedingungen, unter denen es sich allererst artikuliert. Medien bestimmen unsere Rechtslage: "Eine Aktentechnik zieht jeweils eine bestimmte Form und bestimmte Instanzen des Rechts nach sich. Eine andere Bindungsart, eine neue Aufschreibeform, ein geänderter Aufbewahrungsmodus, eine Umstellung bei der Datenerhebung wirken sich auf die Fassung des Rechts aus. Erst in der diachronen Beschreibung differenziert sich also die Rede vom Recht in seine jeweiligen Versionen ${ }^{19}$

Weil Akten und Recht sich gegenseitig definieren, beginnt Vismanns Geschichte des Rechts auch nicht mit dem Übergang von Mündlichkeit zur Schriftlichkeit, sondern mit pragmatischen oder administrativen Schriftformen, mit Gebrauchstexten des Rechts und der Verwaltung also, und das heißt: mit Akten. Nicht die Gründe, die Rechtskulturen $\mathrm{zu}$ schriftlichen Aufzeichnungen bewogen haben mögen, sind in dieser Geschichte entscheidend, sondern die Art und Weise, in der diese administrativen Schriftformen funktionieren, "gerade insofern sie nicht der Logik der gesprochenen Sprache unterliegen ${ }^{20}{ }^{20}$ In den Historischen Hilfswissenschaften, die Texte nicht lesen, sondern angucken und seit dem 19. Jahrhundert medientechnische Textforschung im Dienste historiografischer Sachforschung betreiben, erkennt
Mit Derrida spricht sie von einer Grammatologie der Akten, die nicht den Inhalt, sondern Medialität, Materialität und Funktionalität von Akten untersucht, sowie Akte der Übertragung, Speicherung und des Cancellierens, die nicht nur die Geschichte des Rechts ausmachen, sondern auch an der Formierung derjenigen Entitäten mitwirken, auf denen das Recht beruht: Wahrheit, Staat, Subjekt. Nicht Akten und Akteninhalte bestimmter Behörden interessieren Vismann deshalb, sondern »die Anteile, die staatlich geführte Akten an der Herausbildung von Wahrheitsformen, Staatskonzepten und Subjektvorstellungen in der Geschichte des Abendlands haben ${ }^{21}{ }^{21}$

Dabei sind die Zeiträume, die Vismann in den Blick nimmt, sehr groß, die Perspektive ist mikroskopisch und das Abendland vor allem preußisch. "Akten" erzählt von mehr als 2000 Jahren Rechts- und Verwaltungsgeschichte: Vismann rekonstruiert die von Akten getriebenen Veränderungen der Dispositive des Rechts vom Imperium Romanum und den Kanzleien der Kaiserzeit über den Codex Iustinianus, die Register des Stauferkaisers Friedrichs II. und die preußischen Verwaltungsreformen bis zur "Aktenmäßigkeit der Verwaltung " (Max Weber) in der Weimarer und dem Stasi-Unterlagen-Gesetz der wiedervereinigten Bundesrepublik. Dass Vismann für dieses Unterfangen nicht selbst aus dem Archiv schöpft, sondern sich in Abhängigkeit von älteren historischen Forschungen begibt, hat seinen Preis: So reproduziert sie die Muster dieser Literatur, die im Dienste einer großen nationalen Erzählung viel Gewicht auf das Heilige Römische Reich Deutscher Nation legte, vom Kirchenstaat und dem französischen Königshof aber schwieg. Umgekehrt gilt allerdings dasselbe: Jacob Solls archivgesättigter Meistererzählung über Jean-Baptiste Colberts Erfindung des modernen Staates von 2009 etwa hätte es gutgetan, hätte ihr Autor einen Blick in die englische Übersetzung der "Akten « geworfen. ${ }^{22}$

Der Größe des Untersuchungszeitraums, die bisweilen eher großzügige historische Argumentationen begünstigt, setzt Vismann jedoch immer wieder die Beschreibungsgenauigkeit der Historischen Hilfswissenschaften entgegen. Darin liegt eine der größten Stärken dieses Buches: Wenn Vismanns Thesenbildung sich aus großen historischen Linien ebenso wie aus einer Aufmerksamkeit für paläografische 
und diplomatische Details ergibt, werden Formulare, Register oder Tabellen - nicht unbedingt die ersten Objekte historischen Begehrens - zu welthaltigen Dokumenten und die an ihnen betriebene Empirie theoriefähig. Die genaue Beschreibung solcher Papiertechnologien und die strenge Verknüpfung ihrer Veränderung mit jener von Aktendispositiven und Rechtssystemen sorgt dafür, dass das medientechnische Apriori nicht einfach Suggestion oder Behauptung bleibt.

\section{Akten, Files}

Wenn bei Vismann vermeintlich strockene Verwaltungsgeschichte $\mathrm{zu}$ Theorie wird, die zu lesen ein Vergnügen ist, liegt das auch an der Sprache, in der das Buch geschrieben ist. "Akten" ist, anders als manches Lob es in den Rezensionen nahelegte, keine leichte Lektüre. Aber Wissenschaft ist nun einmal Arbeit und sstilistische Eleganz`vielleicht kein falsches Wort für das Geschick, mit dem Cornelia Vismann, »denkbar komplexe Gedankengänge, in denen sich die unablässige Metamorphose der Akten reflektiert, in eine aphoristisch zugespitzte Formulierung auslaufen [lässt], die dafür sorgt, dass das ausgebreitete Wissenstableau nicht vor den Augen des Lesers verschwimmt «. ${ }^{23}$

Die englische Übersetzung ist nicht zuletzt auch deshalb ein anderes Buch geworden. Vom Medienwissenschaftler Geoffrey Winthrop-Young übersetzt sind die "Akten« 2008 als »Files: Law and Media Technology“ bei Stanford University Press erschienen und dem Andenken des 2007 verstorbenen Historikers Raul Hilberg gewidmet. Der englische Text verzichtet dabei auf viele empirische Details des Originals und sogar auf ganze Unterkapitel, wie etwa auf jenes über die Tabellen der Polizeywissenschaft des 18. Jahrhunderts. Als Teil des Theorieimportunternehmens "German Media Theory“ stärkt die so gekürzte Übersetzung den theoretischen Charakter der Studie. Was der Rezeption des Textes in der englischsprachigen Medienwissenschaft zuträglich gewesen sein dürfte, scheint Archivaren und Historikerinnen die Lektüre verleidet oder zumindest erschwert zu haben. ${ }^{24}$

Enttäuschung über eine ausgebliebene Rezeption der "Akten« war auch eine der Reaktionen auf Cornelia
Vismanns Tod im August 2010. In einem Nachruf, den der Rechtstheoretiker Rainer Maria Kiesow für die "Kritische Justiz« verfasste, vermischt sich die Wut auf den viel $\mathrm{zu}$ frühen Tod mit der auf die Disziplin, der er vorwirft, eine »außergewöhnliche, intellektuelle, professionell und akademisch durchqualifizierte Juristin [...] bis zum Ende ordnungsgemäß betreut und dann zu den Kulturwissenschaftlern abgeschoben « $\mathrm{zu}$ haben. ${ }^{25}$ Die juristische Dissertation ist $\mathrm{zu}$ einem kultur- und medienwissenschaftlichen Standardwerk geworden, während die Rechtsgeschichte ohne "Akten « auszukommen glaubt. Obgleich man zu Kiesows Befund, dass die Verbreitung von Vismanns Schriften "allenfalls an [den] Rändern“ des rechtswissenschaftlichen Diskurses stattfand, inzwischen ergänzen kann, dass sie von einer Richterin am Bundesverfassungsgericht zur Lektüre empfohlen werden. ${ }^{26}$ Es ist wahrscheinlich, dass nicht alle, die Vismanns "Akten" zitieren, sie gelesen haben. Aber das, sagt man, ist das Schicksal von Klassikern.

\section{The Shock of old Paper}

So klar und umfassend, wie in dieser Theorie angelegt, lösen Medien einander nicht ab. Zwar ist es schwieriger geworden, die Steuererklärung auf Papier einzureichen, aber von einem Ende des Papierzeitalters wird kaum sprechen wollen, wer seit Erscheinen von Vismanns Buch auf irgendeiner Behörde war. Dass ein deutscher Geheimdienst Akten schreddert (und nicht Dateien löscht), nachdem die Morde des Nationalsozialistischen Untergrunds als solche bekannt wurden, bestätigt auf zynische Weise die papierne Wahrheit des Schriftlichkeitsgrundsatzes, der auch auf dem Buchdeckel der "Akten" steht: "Quod non est in actis non est in mundo".

Die Wissenschafts- und Technikgeschichte hat der Erfindungsbegeisterung vieler Medien- und Technikgeschichten in den letzten Jahren zwei alternative Konzepte entgegengestellt: die Geschichte der Nutzerinnen und Nutzer und eine Fokussierung auf Störungen. ${ }^{27}$ An Erstere könnte auch eine Rechtsund Verwaltungsgeschichte anschließen, die weniger nach dem Anlegen und Verwahren von Akten fragt als danach, wie Leute in der Begegnung mit den Medien 
des Rechts zu Rechtssubjekten werden. Sie ist in den beiden postum erschienen Büchern Cornelia Vismanns bereits angelegt. Das kurz vor ihrem Tod fertiggestellte "Medien der Rechtsprechung" (Frankfurt am Main 2011) handelt von der theatralen Dimension des Gerichts und lehrt einiges über die mediale Zurichtung von Subjekten vor Gericht. Da Recht eher angewandt oder in Anspruch genommen als genutzt wird, wäre eine auf Klägerinnen, Beklagte, Bürger oder Beamte fokussierende Rechtsgeschichte eine Geschichte der Rechtsanwendung im weiteren Sinne, die papierne Anachronismen ebenso in den Blick nimmt wie die Möglichkeit, nicht nur mit Gesetzen, sondern auch mit Formularen - und mit Akten! - Schindluder zu treiben. ${ }^{28}$

Das fiele auch in den Bereich einer Rechts- und Verwaltungsgeschichte von Störfällen, die nicht nur Disziplinierungs- und also Erfolgsgeschichten, sondern auch von administrativem Scheitern erzählen. Vielleicht wäre heute wieder Berlin der geeignete Ort, um diese Geschichte zu schreiben - nicht so sehr als intellektuelles Milieu wie um die Jahrtausendwende, aber doch als Erfahrungsraum, der einen Anfangsverdacht für eine andere Geschichte der Bürokratie liefert: dass Verwaltungen funktionieren, ist nicht die Regel, sondern die Ausnahme. 
1 Cornelia Vismann, zitiert nach Markus Mohr: Wir sehen uns. Cornelia Vismann in memoriam, in: Ästhetik \& Kommunikation 47 (2017), S. 81.

2 Cornelia Vismann: Akten. Medientechnik und Recht, Frankfurt am Main 2000, S. 335.

3 Vismann: Akten, S. 12.

4 Vismann: Akten, S. 12.

5 Valentin Groebner: Zu den Akten, in: Neue Zürcher Zeitung (14.02.2001).

6 Mario Wimmer: Rezension zu Cornelia Vismann. Akten. Medientechnik und Recht, in: Traverse 18/2 (2011), S. 176.

7 Friedrich Kittler: Bochum, Sommersemester 1988, in: Zeitschrift für Ideengeschichte 6/1 (2012), S. 98.

8 Friedrich Kittler: Es gibt keine Software, in: Friedrich Kittler: Draculas Vermächtnis. Technische Schriften, Leipzig 1993, S. 225.

9 Vismann: Akten, S. 19.

10 Friedrich Kittler: Einleitung, in: Friedrich Kittler (Hg.): Austreibung des Geistes aus den Geisteswissenschaften. Programme des Poststrukturalismus, Paderborn 1980, S. 12.

11 Arndt Niebisch: Close Writing. Friedrich Kittler und die Digital Humanities, in: Metaphora 1: Was waren Aufschreibesysteme? IV, S. 3, online: https://metaphora.univie.ac.at/volume1-niebisch.pdf, (16.05.2019); Vismann: Akten, S. 19.

12 Vismann: Akten, S. 336.

13 Ute Holl: Medientheorie (oder, und, trotz) Kulturtechnikforschung, in: Texte zur Kunst 98 (2015), S. 81.

14 Vismann: Akten, S. 7.

15 Erhard Schüttpelz: Was ist eine Akte?, in: NCCR Mediality Newsletter 7 (2012), S. 5.

16 Vismann: Akten, S. 135.

17 Vismann: Akten, S. 7, 9

18 Vismann: Akten, S. 9.

19 Vismann: Akten, S. 9.

20 Vismann: Akten, S. 19.

21 Vismann: Akten, S. 9-10.

22 Cornelia Vismann: Files. Law and Media Technology, Stanford 2008; Jacob Soll: The Information Master. Jean-Baptiste Colbert's Secret State Intelligence System, Ann Arbor 2009. Siehe zur Kritik am Borussianismus der »Akten«: Groebner: Zu den Akten.

23 Friedrich Balke: Wer kann das alles zusammenhalten!, in: die tageszeitung (9.12.2000).

24 Geoffrey Yeo: A Review of 'Files: Law and Media Technology`, in: Journal of Archival Organization 7/4 (2009), S. 222-223; Wimmer: Rezension, S. 177.

25 Rainer Maria Kiesow: Cornelia Vismann. 26. Mai 1961-28. August 2010, in: Kritische Justiz 43/4 (2010), S. 373.

26 Kiesow: Cornelia Vismann, S. 327; Susanne Baer: Vom Gericht zum Tribunal?, in: die tageszeitung (16.06.2011).

27 Siehe etwa David Edgerton: The Shock of the Old. Technology and Global History since 1900, Oxford 2007; Thomas Bäumler / Benjamin Bühler / Stefan Rieger (Hg.): Nicht Fisch - nicht Fleisch. Ordnungssysteme und ihre Störfälle, Zürich 2011.

28 Zumindest Ersteres ist wiederum in einem späteren Text Vismanns theoretisch erfasst, wenn sie "zwischen Personen, die de jure souverän handeln, und Kulturtechniken, die de facto das Vorgehen bestimmten« unterscheidet. Siehe Cornelia Vismann: Kulturtechniken und Souveränität, in: Cornelia Vismann: Das Recht und seine Mittel, hg. von Markus Krajewski / Fabian Steinhauer, Frankfurt am Main 2012, S. 446. 


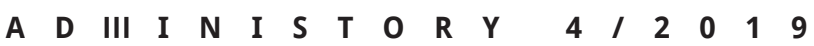

\section{Abstract}

This Relektüre revisits Cornelia Vismann's book Files: Law and Media Technology almost 20 years after its initial publication. Situating the book in the media historical and intellectual constellations in which it originally appeared, this article provides an overview of Vismann's approach to legal and administrative history and sheds light on the book's reception.

\section{About the Author}

Felix Lüttge is a postdoctoral research associate and lecturer (Wissenschaftlicher Assistent) in the Department of Media Studies at the University of Basel. His research focusses on the media history of knowledge and science and on the history and theory of cultural techniques. His first book Auf den Spuren des Wals. Geographien des Lebens im 19. Jahrhundert is forthcoming with Wallstein in 2020. 